\title{
Proposing a new strategy of combining machine learning with controlling cross-transmission of multi-drug resistant organisms of ICU
}

qian zou

Shanghai Jiao Tong University School of Medicine https://orcid.org/0000-0002-8785-306X

Fei Kaihong

Intensive Care Unit, Shanghai General Hospital

Kang Mei

Clinical Research Center, Shanghai General Hospital

Li Xianchen

Clinical Research Center, Shanghai General Hopspital

Ding Mengyuan

Shanghai Jiaotong University School of Medicine

Geng Miaomiao

Shanghai Jiao Tong University School of Medicine

Zhu Yanhong ( $\nabla$ yanhongzhu2010@163.com )

Research

Keywords: multi-drug resistant, intensive care unit, cross-transmission, machine learning, precaution, predictive

Posted Date: July 2nd, 2020

DOl: https://doi.org/10.21203/rs.3.rs-39568/v1

License: @ (i) This work is licensed under a Creative Commons Attribution 4.0 International License. Read Full License 


\section{Abstract \\ Background}

Multi-drug resistant organisms (MDROs) has become a global threat to public health. MDROs are normally transmitted from patients to patients via the hands of healthcare workers (HCWs). The key management of MDROs is control dissemination as sooner as possible.

\section{Method:}

We established a predictive rule based simply on experiences, and according to the result of this predictive rule we take a series of precautions of a general intensive care unit (ICU) from January 1, 2018, to December 31, 2019, only in one ward experimentally. In this study, we aim to assess the efficiency of the routine care practice which include pre-discrimination by the predictive rule and sequent precautions by doing difference comparisons.

\section{Results}

After comparing two wards in the hospital expenses and length of ICU stay, there are no statistical differences. Precautions contribute to the association between room number and status of MDROs infection/ colonization $(p=0.033)$, and infection/colonization rate of MDROs is different statistically between two $\operatorname{wards}(p=0.006)$.

\section{Conclusion}

the routine care practice had controlled the cross-transmission of MDROs in some extent. Future, studies can engage in updating the predictive model based on big data and referred to experts' experiences and adopt more efficient precautions for strengthening the transmission efficiency.

\section{Background}

Multi-drug resistant organisms (MDROs) have become a global threat for public health. In American, more than 700,000 healthcare-associated infections occur annually, and many caused by antibiotic-resistant bacteria [1]. According to statistics from the China Antimicrobial Resistance Surveillance System (CARSS), Methicillin-resistant Staphylococcus aureus (MRSAs) accounted for $30.9 \%$ of S. aureus isolates and Carbapenem-resistant Acinetobacter baumannii compose $56.1 \%$ of baumannii isolates, respectively, in 2018 [2]. D.J. Morgan and Sarah S. Jackson found that when Healthcare Workers (HCWs) contact patients with MDR bacteria they frequently contaminate their protective gowns and gloves [3][4]. Multi-drug resistant organisms are normally transmitted from patients to patients via the hands of HCWs [5]. MDROs infection/colonization leads to clinical consequences: intensive care unit (ICU) length of stay, limiting treatment options, increased risk of therapeutic failure, and higher mortality and costs [6-9]. The key step of decreasing the dissemination rate of MDROs is to cut off the spread chain of people to people.

In China, experts advised that patients should have positive screening once admitted to ICU to find out whether they have colonized MDROs or not. It aims to take sequence precautions, like isolation, to prevent nosocomial cross-transmission of MDROs as early as possible, since solation measures are recommended to reduce transmission of MDR bacteria in the ICU [5][10]. But growing bacterial cultures and doing a susceptibility test take a few days in China, usually, it is too late to benefit from isolation.

In this article, we introduce an exploratory experiment carried out in our hospital from January 1, 2018, to now (March 2020). The experiment is about applying an elementary predictive model to pre-discriminate the status of patients before the clinical culture testing results came out and then giving interventions firstly. we also will evaluate the effect of this experiment in this article and raise some opinions in the management of MDROs in ICU.

\section{Method}

\section{Hospital setting}

This study was undertaken in a general ICU (including ward 2A and 2B) of 32 beds (2A has 12 beds), a university-affiliated hospital, located in Shanghai, China. It is a tertiary hospital, which is divided into two branches: the north one and south one. The experiment practiced in ward $2 \mathrm{~B}$ of the south one only, because the author (Fei Kaihong) is the head nurse of the south branch's ICU(2B). Patients admitted to ICU are arranged to two wards randomly. In 2B, there have 3 quadruple rooms (bed number from 11 to 14, 19 to 22 and 23 to 26), 2 double rooms (bed number include 15,16,17 and 18), and 6 single rooms.

\section{The exploratory experiment}

From January 1, 2018, to December 31, 2019, the head nurse of the 2B part applied an elementary predictive rule to pre-discriminate the status of MDROs colonization/infection. There are eight relevant factors in the predictive rule. Meeting one clause, HCWs judged that the patient has a very high risk for colonization/infection-supposing to have colonized/infected MDROs. The detail of this rule shows in table 1. If the status assumed to be colonized/ infected with MDROs, the HCWs will strengthen hand hygiene and wear isolation gowns, before and after contacting patients. If circumstances permitted, everyone predicted positive with MDROs should be isolated into a single room or arrange infected or colonized patients separate from other patients otherwise. This preintervention will continue until acquiring the clinical culture results. 
Table 1 an elementary predictive rule applied in ICU ward 2B

\begin{tabular}{|l|}
\hline Clauses \\
\hline Antibiotic treatment before admitted to ICU \\
\hline Hospital stays longer than 2 weeks \\
\hline Have underlying health problems and older than 60 \\
\hline Have done invasive procedures \\
\hline treated with immunosuppressive agents \\
\hline Known to have colonized with MDROs \\
\hline Sepsis or organ failure before ICU admission \\
\hline Admitted to ICU abruptly \\
\hline
\end{tabular}

\section{Data collection}

Because this is a retrospective sharing of ICU clinical practice, data dug out from the hospital electronic file and CARSS, retrospectively, to assess the efficiency and benefit of this routine ICU caring. Data included age, gender, ward (2A or 2B?), hospitalization expenses(on April 1, 2020, 1 CNY=0.1408 \$), admission date, discharge data, length of hospital stay, bed number and status of MDROs colonization/infection from screening results from January 1, 2018, to December 31, 2019. To analyze the length of clinical culture testing, we exported the date of sample submission and the date of acquiring testing results from January 1, 2018, to December 31, 2019, of the whole ICU.

\section{Statistical analysis}

Quantitative variables, normally distributed, are expressed as mean (standard deviation), or median (interquartile range) otherwise. Categorical variables described by number (percentage). Before statistics, quantitative variables use histograms and the Shapiro-Wilk test to assess the normality of distribution. Quantitative variables were examined using Student's t-tests for normally distributed variables and Wilcoxon rank-sum tests for non-normally distributed variables. Chi-square tests were used for categorical variables. All reported P values are 2-tailed, with a P-value $<0.05$ considered statistically significant. Statistical analyses were performed using SPSS, version 25.0.

We performed differences comparison between 2A and 2B. Variables contain age, gender, hospitalization expenses, ICU length of stay, and status of MDROs colonization/ infection. We examined age as a categorical variable. Age categories used were $\geq 65$ years and $<65$ years. Hospitalization expenses converted to US dollars (refer to the China-US exchange rate on April 1, 2020, $1 \mathrm{CNY}=0.1408$ ). Hospitalization expenses are divided into two groups: $\geq 6500 \$$ and $<6500 \$$. Because patients in ICU are normally had tens of days of admission length, 14 days serving as a cutoff point, ICU length of stay is examined as a categorical variable, too.

\section{Results}

A total of six hundred thirty-six patients were admitted in the ICU setting among which 198 comes from 2A and 438 stems from 2B. Patients colonized/ infected with MDROs are 68 , counting for $10.69 \%$ of whole specimens. Of the whole 636 patients, males were 389 and females were 247 . Characteristics summarized in Table 2.

\section{The exploratory experiment benefit evaluation}

Because the 2A ward did not have the experimental practices, we do performance comparisons between $2 \mathrm{~A}$, and $2 \mathrm{~B}$ in hospitalization expenses, ICU length of stay and the real MDROs infection rate. The characteristics of patients of two wards and performance comparison of the exploratory experiment are showing in table 2. We can see that in two wards, age and gender have no statistical difference, $p=0.812$, and $p=0.212$, respectively. As performances comparison index of the exploratory experiment, for example, hospitalization expenses and ICU length of stay, have no difference statistically, too, $p=0.219$ and $p=0.386$, respectively. However, there do have a statistical difference in MDROs infection status of these two ward's patients $(p=0.006,<0.05)$.

We also analyzed the association between room number and MDROs infection status. It is noteworthy that we excluded single rooms in this association analyzation because single rooms are prepared exactly for infected patients to isolated with uninfected patients, there has a strong relevance between the single rooms and infected status-causing bias of our result. The result of the association showed in table 3 , we could see that room number is associated with MDROs infection status, $p=0.033$. It suggested that the exploratory experimental routine practices maybe truly discriminated against colonized/ infected people and the intervention curbed cross-transmission between rooms, so the infection occurred only in some specific rooms.

Table 2 characteristics of patients of two wards and performance comparison of the exploratory experiment 


\begin{tabular}{|c|c|c|c|c|}
\hline & \multicolumn{2}{|l|}{ Wards } & \multirow[t]{2}{*}{$\square^{2}$} & \multirow[t]{2}{*}{$P$-value } \\
\hline & $2 \mathrm{~A}(\mathrm{~N} 0=$ & $2 \mathrm{~B}(\mathrm{~N} 1=438)$ & & \\
\hline age & & & 0.057 & 0.812 \\
\hline$\geq 65$ & $72(36.4 \%)$ & $155(35.4 \%)$ & & \\
\hline$<65$ & $126(63.6 \%)$ & $283(64.6 \%)$ & & \\
\hline gender & & & 1.558 & 0.212 \\
\hline male & $114(57.6 \%)$ & $275(62.8 \%)$ & & \\
\hline female & $84(42.4 \%)$ & $163(37.2 \%)$ & & \\
\hline hospitalization expenses & & & 1.511 & 0.219 \\
\hline$\geq 6500, \$$ & $75(37.9 \%)$ & $144(32.9 \%)$ & & \\
\hline$<6500, \$$ & $123(62.1 \%)$ & $294(67.1 \%)$ & & \\
\hline ICU length of stay & & & 0.753 & 0.386 \\
\hline$\geq 14$, days, & $51(25.8 \%)$ & $99(22.6 \%)$ & & \\
\hline$<14$, days, & $147(74.2 \%)$ & $339(77.4 \%)$ & & \\
\hline MDROs infection status & & & 7.422 & 0.006 \\
\hline infected & $31(15.7 \%)$ & $37(8.4 \%)$ & & \\
\hline uninfected & $167(84.3 \%)$ & $401(91.6 \%)$ & & \\
\hline
\end{tabular}

Table 3 the association analysis between room number and MDROs infection status in ward 2B

\begin{tabular}{|lllll|}
\hline & \multicolumn{2}{c}{ MDROs infection status } & $\mathbb{Z}^{2}$ & $P$-value \\
\cline { 2 - 4 } & infected & uninfected & & \\
\hline Room number & & & 10.472 & 0.033 \\
\hline 1 & $4(28.6 \%)$ & $54(20.9 \%)$ & & \\
\hline 3 & $6(42.9 \%)$ & $41(15.8 \%)$ & & \\
\hline 4 & $3(21.4 \%)$ & $49(19.0 \%)$ & \\
\hline 5 & $0(0.0 \%)$ & $56(21.7 \%)$ & \\
\hline
\end{tabular}

\section{The length of clinical culture testing}

We exported the date of sample submission and minus the date of acquiring testing results to representing the length of clinical culture testing. From January 1, 2018, to December 31, 2019, we found 1065 records of ICU in the hospital of the north branch. We found one outlier during statistical describing. The data of clinical culture testing days does not conform to the normal distribution, $p<0.05$ (Shapiro-Wilk test). The median is 3.40 and the interquartile range is 1.96 with the outlier being excluded.

\section{Discussion}

As for assessing the efficiency of an index or risk score identifying MDROs status at hospital admission, researchers used additional isolation days, costs of additional isolation, percentages of patients with ICU-acquired infection of MDROs, ICU length of stay, ICU mortality and calibration of a predictive model by the area-under-the-curve (AUC) usually[11][12][13][14]. In this study, we did not find statistical differences between hospital expenses and length of ICU stay. We analyzed the difference in age and gender between two groups only. But there are lots of factors associated with MDROs status, like invasive operation, having chronic renal disease, APACHE $\geq 15$, previous hospital stay $\geq 10$ days, emergency surgery, and so on[15][16].Since these factors can influence hospital expenses and length of ICU stay, so we got no difference between the two groups. We also think the perfect endpoint of the benefit of precaution is the dissemination rate of MDRO. In our experiment, only patients identified by the predictive rule as MDROs infection/colonization having screened the bacteria, so we cannot calculate the cross-transmission rate and assess the predictive rule's discrimination and calibration. A study conducted in Singapore assessed the predictive model of MDROs by area-under-the-curve (AUC), evaluating a not bad result. Because it is a one center study, we could not use that model in our hospital, and the situation is different between countries. 
In our study, there does have an association between room number and status. In our knowledge, this is the first study to analyze the association between room number/ bed number and MDROs infection status. The result suggested that the pre-intervention work and it is possible to control cross-transmission of multi-drug resistant pathogens. Discriminating infection or colonization is just a first step. The purpose is to control cross-transmission between patients. A study reported percentages of cross-transmission in ICU ranging from 23-53\% due to patients' contacts [17]. We know that colonized or infected patients, contaminated hands or grows of HCWs, visitors, and environment all could be reservoirs of MDROs. The WHO suggests HCWs should use contact precautions when providing care to patients who are known or suspected to be infected or colonized with MDROs [18]. The key problem is how to know the patient whether he has been infected or colonized nor not as sooner as possible. A guideline-recommended active surveillance contributes to stopping the spread of MDROs by early detection [19]. N. Yamamoto found that rapid intervention based on a rapid molecular diagnostic assay contributed to reduing nosocomial transmission of carbapenem-resistant Acinetobacter baumannii in ICUs [20]. In this study, we analyzed the length of clinical culture testing, it takes 3.4 days averagely in our hospital. So, we boldly speculated that active surveillance and molecular diagnostic assay still time-consuming. In this article, we want to highlight the necessity of combining machine learning with medicine and the golden time for precaution is patient admission time. Based on big data to figure out predictive models of MDROs is just a kind of combination. A study conducted in China came up with using American Thoracic Society Guidelines to predict the status of MDROs infection or colonization, but it failed [21].

The method of combining machine learning with MDROs status' diagnosis in China is in the initial stage. We innovatively bring forward that using a predictive rule to pre-discriminate the status of MDROs infection or colonization and then guide accurate intervention on admission. Still, there are some limitations: Firstly, because this is a clinical practice, we could not require every patient to screen on admission. Secondly, the sample size is not big enough, the number of dead in ICU is too small. Third, the baseline information is limited, it cannot control the basic situations of patients between patients in 2A and $2 \mathrm{~B}$, then, the result of the comparison between two groups was lack of evidence. Most importantly, the predictive model was established empirically. Future we need to work on the perfection of the new model both referring to machine learning and consulting experts in nosocomial infection fields. To evaluate the new model, we should conduct a prospective cohort study or before-and-after study. Discrimination and calibration are essential indexes to evaluate the efficiency of the predictive model. Data on baseline information of patients should collect prospectively as detailed as possible.

\section{Conclusion}

In this study, we found that the routine care practice including predicting the status of MDROs and sequent precautions had controlled the cross-transmission of MDROs to some extent. It throws light on that this is a feasible strategy to overcome the challenge of controlling the transmission of nosocomial infection. Future, studies can engage in updating the predictive model based on big data and referred to experts' experiences and adopt more efficient precautions for strengthening the transmission efficiency.

\section{Declarations}

\section{Funding}

This work was supported by General Program on National Natural Science Foundation of China (71974127) and Key Program on Hospital Management of China Hospital Development Institute of Shanghai JiaoTong University (CHDI-2018-A-04).

\section{Availability of data and materials}

Authors can confirm that all relevant data are included in the article.

\section{Authors' contributions}

ZQ and FK contributed equally to the writing of this article. ZQ and FK involved in the preparation of the proposal and study design, participated in data collection, data entry and data analysis as manuscript preparation. KM and LX contributed to acquisition of data, conception and design of this study. DM and GM contributed to acquisition, analysis and interpretation of data. ZY contributed to conception and design of this study, have been involved in revising the manuscript critically for important intellectual content. All authors read and approved the final manuscript.

\section{Ethics approval and consent to participate}

Not applicable.

\section{Consent for publication}

Not applicable.

\section{Competing interests}

The author declare that they have no competing interests.

\section{Acknowledgements}

Not applicable.

\section{References}


1. Magill SS, Edwards JR, Bamberg W, Beldavs ZG, Dumyati G, Kainer MA, Lynfield R, Maloney M, McAllister-Hollod L, Nadle J, Ray SM, Thompson DL, Wilson LE, Fridkin SK. Infections Emerging Infections Program Healthcare-Associated, Team Antimicrobial Use Prevalence Survey (2014) Multistate point-prevalence survey of health care-associated infections. N Engl J Med 370(13):1198-1208. doi:10.1056/NEJMoa1306801.

2. Committee of Experts on Rational Drug Use of the National Health and Family Planning Commission of the P.R.CHINA, China Antimicrobial Resistance Surveillance System. 2018 National Bacterial Resistance Surveillance Report. Chinese Journal of Rational Drug Use 2020; 17(1):1-10. doi:10. 3969/j.issn.2096-3327.2020.1.001 Available at: https://kns.cnki.net/KXReader/Detail? TIMESTAMP=637287087080889901\&DBCODE=CJFQ\&TABLEName=CJFDLAST2020\&FileName=ZYYS202001001\&RESULT =1\&SIGN=Dvhs\%2fZ1hBE9IU

3. Morgan DJ, Rogawski E, Thom KA, Johnson JK, Perencevich EN, Shardell M, Leekha S, Harris AD. Transfer of multidrug-resistant bacteria to healthcare workers' gloves and gowns after patient contact increases with environmental contamination. Crit Care Med. 2012;40:1045-51. doi:10.1097/CCM.0b013e31823bc7c8.

4. Jackson SS, Thom KA, Magder LS, Stafford KA, Kristie JJ, Miller LG, et al. Patient contact is the main risk factor for vancomycin-resistant enterococcus contamination of healthcare workers' gloves and gowns in the intensive care unit. Infection Control \& Hospital Epidemiology (2018), 1-5.

5. Strich JR, Palmore TN. Preventing Transmission of Multidrug-Resistant Pathogens in the Intensive Care Unit. Infectious Disease Clinics of North America 2017(31):535-550.

6. Roberts RR, Hota B, Ahmad I, et al. Hospital and societal costs of antimicrobial resistant infections in a Chicago teaching hospital: implications for antibiotic stewardship. Clin Infect Dis. 2009;49(8):1175-84.

7. Melsen WG, Rovers MM, Groenwold RHH, Bergmans DCJJ, Camus C, Bauer TT, et al. Attributable mortality of ventilator-associated pneumonia: A metaanalysis of individual patient data from randomised prevention studies. Lancet Infect Dis. 2013;13:665-71.

8. Bassetti M, De Waele JJ, Eggimann P, Garnacho-Montero J, Kahlmeter G, Menichetti F, et al. Preventive and therapeutic strategies in critically ill patients with highly resistant bacteria. Intensive Care Med. 2015;41:776-95.

9. Masse J, Elkalioubie A, Blazejewski C, et al. Colonization pressure as a risk factor of ICU-acquired multidrug resistant bacteria: a prospective observational study. Eur J Clin Microbiol Infect Dis. 2017;36(5):797-805.

10. Tacconelli E, Cataldo MA, Dancer SJ, De Angelis G, Falcone M, Frank U, et al. ESCMID guidelines for the management of the infection control measures to reduce transmission of multidrug-resistant Gram-negative bacteria in hospitalized patients. Clin Microbiol Infect. 2014;20:1-55.

11. Marjan Wassenberg J, Kluytmans S, Erdkamp, et al. Costs and benefits of rapid screening of methicillin-resistant Staphylococcus aureus carriage in intensive care units: a prospective multicenter study. Critical Care, 2012:2-8.

12. Ledoux G, Six S, Lawson R, Labreuche J, Blazejewski C, Wallet F, et al. Impact of a targeted isolation strategy at intensive-care-unit-admission on intensive-care unit-acquired infection related to multidrug-resistant bacteria: a prospective uncontrolled before-after study. Clinical Microbiology Infection. 2016;22:888, e11-888.e18.

13. Djibré M, Fedun S, LeGuen P, Vimont S, Hafiani M, Fulgencio J-P, et al. Universal versus targeted additional contact precautions for multidrug-resistant organism carriage for patients admitted to an intensive care unit. Am J Infect Control. 2017;45:728-34.

14. Vasudevan A, Mukhopadhyay A, Li J, Yuen EGY, Tambyah PA. A prediction tool for nosocomial multi-drug Resistant Gram-Negative Bacilli infections in critically ill patients-prospective observational study. BMC Infect Dis. 2014;14:615.

15. Kiddee A, Assawatheptawee K, Na-Udom A, Boonsawang P, Treebupachatsakul P, Walsh TR, Niumsup PR. Risk Factors for Extended-Spectrum $\beta$ Lactamase-Producing Enterobacteriaceae Carriage in Patients Admitted to Intensive Care Unit in a Tertiary Care Hospital in Thailand, Microbial drug resistance (Larchmont, N.Y.) 25 (2019) 1182-1190. 10.1089/mdr.2018.0318.

16. Palomar M, Alvarez Lerma F, Catalan M, Uriona S, Nuvials X, Gimeno R, Olaechea P, Aragon C, Gracia MP, Vallverdu M. Risk factors for multiresistant bacterias in critical patients. Are there differences between the previous acquisition or during the stay in ICU? Intensive Care Medicine Experimental 6 (2018). 10.1186/s40635-018-0201-6.

17. Lingaas E, Fagernes M. Development of a method to measure bacterial transfer from hands. J Hosp Infect. 2009;72:43-9.

18. WHO Guidelines on Hand Hygiene in Health Care. First Global Patient Safety Challenge, Clean Care Is Safer Care. Geneva: World Health Organization; 2009. https://apps.who.int/iris/bitstream/handle/10665/44102/9789241597906_eng.pdf?sequence=1.

19. Tacconelli E, Cataldo MA, Dancer SJ, De Angelis G, Falcone M, Frank U, Kahlmeter G, Pan A, Petrosillo N, Rodriguez-Bano J, et al. ESCMID guidelines for the management of the infection control measures to reduce transmission of multidrug-resistant gram-negative bacteria in hospitalized patients. Clin Microbiol Infect. 2014;(20 Suppl): 1:1-55.

20. Yamamoto N, Hamaguchi S, Akeda Y, Santanirand P, Chaihongsa N, et al. Rapid screening and early precautions for carbapenem-resistant Acinetobacter baumannii carriers decreased nosocomial transmission in hospital settings: a quasi-experimental study. Antimicrob Resist Infect Control. $2019 ; 8: 110$. 10.1186/s13756-019-0564-9.

21. Xie J, Ma X, Huang Y, Mo M, Guo F, Yang Y, Qiu H. Value of American Thoracic Society guidelines in predicting infection or colonization with multidrugresistant organisms in critically ill patients. PLoS One. 2014;9:e89687. 10.1371/journal.pone.0089687. 